and it may also allow screening of candidate drugs to treat the lipidoses. (Chen C-S, Patterson MC, Wheatley CL, O'Brien JF, Pagano RE. Broad screening test for sphingolipid-storage diseases. Lancet Sept 11, 1999;354:901-905). (Respond: Dr Richard E Pagano, Mayo Clinic and Foundation, Guggenheim 621-C, 200 First Street SW, Rochester, MN 55905).

COMMENT. This simple fluorescence assay should be helpful in the investigation of children with developmental disorders and progressive neurodegenerative diseases and the exclusion of sphingolipid-storage diseases in patients with atypical or mild variants.

In a commentary by Bryan Winchester, Institute of Child Health, London (Lancet Sept 11, 1999;354:879-880), the method is described as potentially an important advance. He suggests that adaptation of the method to allow measurement of the fluorescence using microtitre-plate technology in whole cells, preferably white blood cells, would simplify the procedure and avoid the delay and cost of culturing fibroblasts.

\title{
AUTOSOMAL DOMINANT JUVENILE AMYOTROPHIC LS
}

The clinical and electrodiagnostic findings in 49 affected family members and neuropathological findings from two autopsies of a Maryland kindred with autosomal dominant juvenile amyotrophic lateral sclerosis (ALS) are reported from Johns Hopkins University and Hospital, Baltimore, MD. The ALS was linked to the chromosome 9q34 region (ALS4). The mean age at onset was 17 years, and patients ranged in age from 12 to 85 years (mean 45 years). Clinically, the majority showed distal weakness and atrophy associated with pyramidal signs and normal sensation. Electrodiagnostic testing in 8 patients showed reduced evoked amplitudes and normal motor and sensory conduction. EMG showed distal chronic partial denervation and reinnervation. Spinal cord tissue was atrophic with loss of anterior horn cells, degeneration of corticospinal tracts, loss of neurons in the dorsal root ganglia, and degeneration of the posterior columns. Motor and sensory roots and peripheral nerves showed axonal loss and diffuse prominent swellings. This family extends the genetic heterogeneity of familial and juvenile ALS. (Rabin BA, Griffin JW, Crain BJ, Scavina M, Chance PF, Cornblath DR. Autosomal dominant juvenile amyotrophic lateral sclerosis. Brain Aug 1999;122:1539-1550). (Respond: Dr David R Cornblath, Pathology 627, Johns Hopkins Hospital, Baltimore, MD 21287).

COMMENT. Juvenile ALS is a chronic motor neuron disease with onset before 25 years and characterized by upper and lower motor neuron dysfunction in the absence of sensory abnormalities or ataxia. A family is described with a slowly progressive, non-fatal, autosomal dominant form of juvenile ALS linked to the chromosome 9q34 (ALS4).

\section{SEIZURE DISORDERS}

\section{BENIGN PARTIAL SEIZURES OF ADOLESCENCE}

Eight patients matching the description of benign partial seizures of adolescence (BPSA), as described by Loiseau et al in 1978, were found among 92 teenagers, including 37 with new-onset focal seizures, enrolled in a prospective first-seizure study at the University of Melbourne, Victoria, Australia. Four of the 8 patients with BPSA were boys and 4 girls, aged 11-17 years. All seizures were Jacksonian in pattern, with a sensory/motor march, 6 were secondarily 
generalized, and 2 had only simple partial seizures before referral. The initial EEG was normal in 6; subsequent sleep deprived EEGs showed focal epileptiform transients in 3. Postictal recordings showed unilateral multifocal epileptiform discharges in one and a single occipital discharge in one. MRI was normal in all patients. Three were treated with carbamazepine for 2 years, and one had seizure recurrence after discontinuing treatment. Three had infrequent simple partial seizures at 2 year follow-up. (King MA, Newton MR, Berkovic SF. Benign partial seizures of adolescence. Epilepsia 1999;40:1244-1247). (Reprints: Dr SF Berkovic, Department of Neurology, Austin and Repatriation Medical Centre, Heidelberg (Melbourne), Victoria 3084, Australia).

COMMENT. Partial seizures of adolescence, characterized by a sensory/motor march, can be idiopathic and relatively benign, and may not invariably require antiepileptic therapy. The authors present a group of patients with so-called "benign partial seizures of adolescence," representing $22 \%$ of newonset focal seizures in teenagers enrolled in a prospective study.

When to start and stop anticonvulsant therapy is a question reviewed by Greenwood RS, Tennison MB (Arch Neurol Sept 1999;56:1073-1077). In general, AEDs should be withheld until after a second seizure and tapered after 2 years without seizures. The risks and benefits are discussed. In practice, the decision must be individualized and a general rule does not apply.

\section{ATTENTION DEFICIT DISORDERS}

\section{SLEEP DISORDERS IN ATTENTION DEFICIT DISORDER}

Parental perceptions of sleep patterns in children presenting at an ADHD clinic in Washington, DC, were assessed prospectively over a 6-month period. Of 108 children diagnosed with ADHD, 46 were taking stimulant medications. Parents of all ADHD patients, 35 controls with psychiatric disorders attending the same clinic, and 84 general pediatric outpatients were given a Sleep Behavior questionnaire, the Achenbach Child Behavior Checklist, and a medical history questionnaire.

Moderate to severe sleep disorders occurred in 22\% of ADHD children, 10\% of psychiatric controls, and $6 \%$ of pediatric controls $(\mathrm{p}<0.05)$. Among ADHD children, insomnia was increased three-fold in patients receiving stimulant therapy. In addition to insomnia, ADHD patients had daytime tiredness, decreased sleep requirement, and frequent awakenings, not associated with parasomnias, enuresis, or respiratory disturbances. (Pearl PL, Stein MA, Broitman M, Efron L, Hamburger E. Sleep disturbances in children with attention deficit hyperactivity disorder. Ann Neurol Sept 1999;46:524; abstract).

COMMENT. Sleep disorders are more common in ADHD children than in other pediatric patients, and are more prevalent and severe in patients treated with stimulant medications. Questions regarding sleep habits are important in the initial and follow-up examinations of children with ADHD. The substitution of an antihypertensive agent, clonidine or Tenex, may be advisable when insomnia is severe. A combination of stimulant and clonidine is not generally advised, because of reported cardiac complications and rare fatalities. See Ped Neur Briefs May 1999;13:40, for a debate regarding methylphenidate and clonidine combination therapy (Swanson JM, Connor DF, Cantwell D. L Am Acad Child Adolesc Psychiatry May 1999;38:614-622). 